\title{
More space
}

\section{As we celebrate the twenty-fifth anniversary of the Hubble Space Telescope, there is plenty to look back on and even more to look forward to.}

In this issue of Nature Physics, we focus on space. Specifically, we look at four space missions past and present, as well at the prospect of quantum technologies in space.

The Hubble Space Telescope (HST) is not the first or even the biggest telescope but it has gripped the public. Its optical images are so vivid and detailed that school children believe the telescope travels to distant galaxies when in fact it follows a low-Earth orbit. The key advantage is that HST lies beyond the attenuating atmosphere and has a suite of instruments to study voids, clusters, supermassive black holes and everything else in between. And its original launch and subsequent servicing by space shuttle have only added to the drama. As HST reaches twenty-five years in space, Luis C. Ho shares his personal experience of the iconic telescope on page 607.

And as Nature Physics goes to press, the New Horizons mission to Pluto and the Kuiper Belt has made all the headlines. Before the fly-by on 14 July, our best images of Pluto were taken by HST in 2002-2003 (upper image). And now, nine years and 4.8 billion $\mathrm{km}$ from launch, we see an icy planet with mountains and troughs (lower image) within a methane and nitrogen atmosphere. The mountains are younger than 100 million years, suggesting that Pluto is geologically active. It is the moon landing of our generation. Indeed, Pluto is two-thirds the size of our moon, and yet it has five moons - two of them unknown before launch. And to put things into perspective, so little sunlight reaches Pluto that the copper-hued surface would appear greyish to a person standing on it.

But not to be dwarfed, the Dawn mission to asteroid Vesta and dwarf planet Ceres in the asteroid belt between Mars and Jupiter is making plenty of discoveries. Currently, the spacecraft is in orbit around Ceres, which may contain water or ice. Bright spots on the surface suggest subsurface ice (or even salt) and require further investigation. William B. McKinnon brings us up to date with both missions to the dwarf planets. As for Vesta, scientists hope to learn more about the giant crater on its southern pole. The impact that created the crater blasted $1 \%$ of Vesta's mass into space, possibly resulting in 5\% of the meteorites that have since arrived at the Earth.
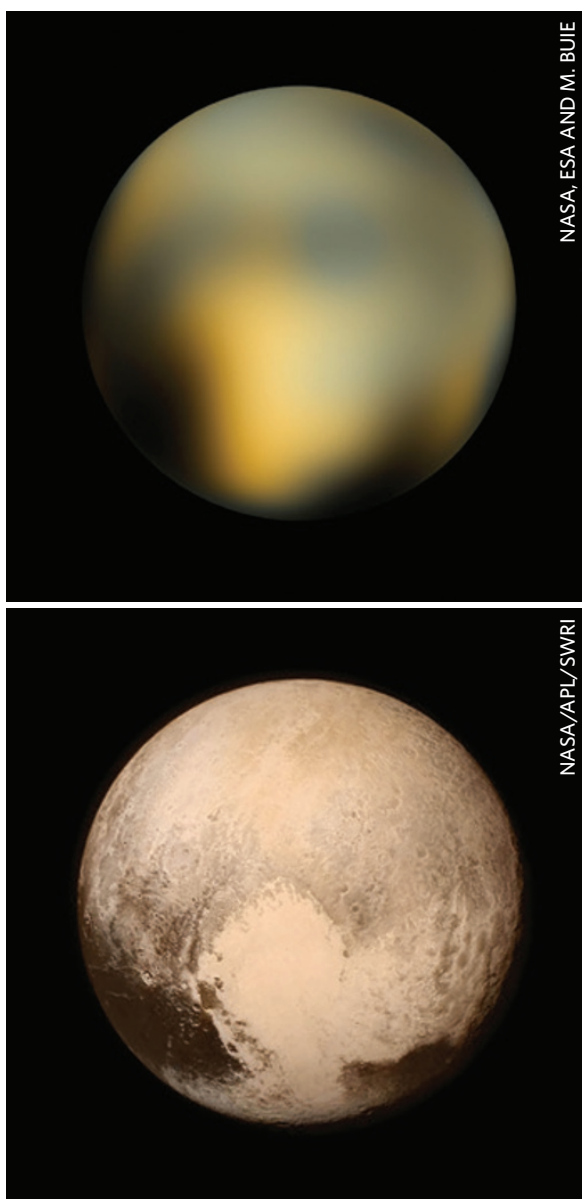

Closer to home, the Magnetospheric MultiScale (MMS) mission, launched earlier this year, is designed to measure Earth's protective magnetosphere, consisting of charged particles that are controlled by the Earth's magnetic field. Interactions with plasma in the solar wind create an asymmetry that is variable. Detailed measurements of the plasma and electric and magnetic fields will help us understand how the magnetic field lines disconnect and reconnect around different plasma cells. This process is known collectively as magnetic reconnection, which releases energy to the magnetosphere. The MMS mission, with four identical spacecraft arranged in a tetrahedron to obtain full threedimensional views, will provide information on the rate of reconnection and its large variations. In the Commentary on page 611 , Thomas Earle Moore, James L. Burch and Roy B. Torbert describe how magnetic reconnection connects our planet to the rest of the Universe.

Later this year, the launch of LISA Pathfinder will bring us closer to detecting gravitational waves in space. It will place two test masses in gravitational free-fall - something that can only be done in space. The instrument suite to control and measure them will establish the limits of laser interferometry at low frequencies and picometre resolution in space, laying the groundwork for the future gravitational wave observatory eLISA, which can be thought of as a Michelson interferometer with an effective arm-length of 1 million kilometres. For the big picture, see Karsten Danzmann's Commentary on page 613 .

Looking even further ahead, Kai Bongs, Michael Holynski and Yeshpal Singh outline plans for sending quantum technologies into space. More than just improving secure communications based on satellites, these applications of quantum physics can also provide more sensitive measurements of global water and climate data. On the more fundamental side, improved data of the gravitational potential can help test theories for unifying quantum gravity and general relativity. Cold atom based interferometry also represents a complementary means of measuring gravitational waves with both Earth and space based observatories.

And finally, a look into the future for a glimpse of our past. Sometime in 2020 or thereabouts - it is difficult to say at this point-HST will shut down and leave its orbit. By 2018, its successor, the James Webb Space Telescope, should be in place. Its near- and mid-infrared capabilities are designed to probe the early Universe, when the first stars and galaxies were formed (about 13.5 billion years ago). Even 400,000 years after the Big Bang, the Universe was a dark place, and we know very little about the first sources of light and how they subsequently shaped galaxies. These ultra-deep surveys will reveal how the Universe emerged from the 'Dark Ages' of cosmic history. 